\title{
On the necessity and feasibility of 3D simulations of steam cracking reactors
}

\section{Supporting Information}

Pieter A. Reyniers ${ }^{1}$, Carl M. Schietekat ${ }^{1}$, David J. Van Cauwenberge ${ }^{1}$, Laurien A. Vandewalle $^{1}$, Kevin M. Van Geem ${ }^{1, *}$, Guy B. Marin ${ }^{1}$

${ }^{1}$ Ghent University, Laboratory for Chemical Technology, Technologiepark 914, 9052 Gent, Belgium.

${ }^{*}$ Corresponding author: Technologiepark 914, 9052 Gent, Belgium;

$\underline{\text { Kevin.VanGeem@UGent.be }}$ 


\section{Comparison of reduced to full network}

The reduced network used in the simulations of the industrial propane cracking reactor was obtained from the full network of Dijkmans et al. ${ }^{1}$ by removal of all species and reactions irrelevant for propane cracking. Important to note is than no kinetic parameters were adjusted to improve the agreement between the reduced and the full network. To assess the validity of the reduction for propane crackiing, 14 isothermal plug flow simulations were performed with both the reduced and the full network at a temperature range from 873 to $1173 \mathrm{~K}$ covering the entire propane conversion range.

Figure S1 A shows that a good agreement is obtained for the conversion as a function of reactor temperature. Also for the hydrogen, methane and ethene yield shown in Figure S1 B, $\mathrm{C}$ and $\mathrm{D}$ respectively, a good agreement between the reduced and the full network is seen.

A

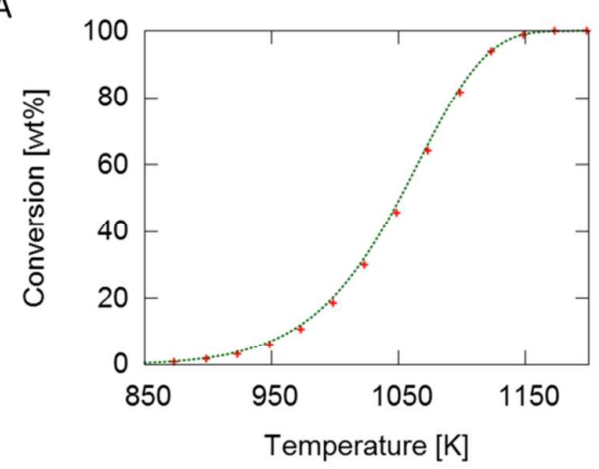

C

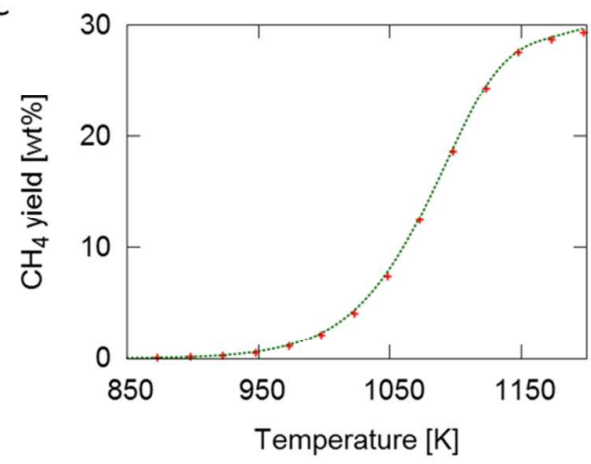

B

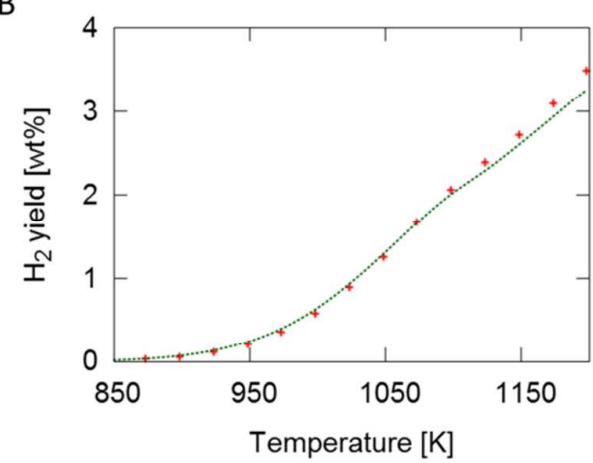

D

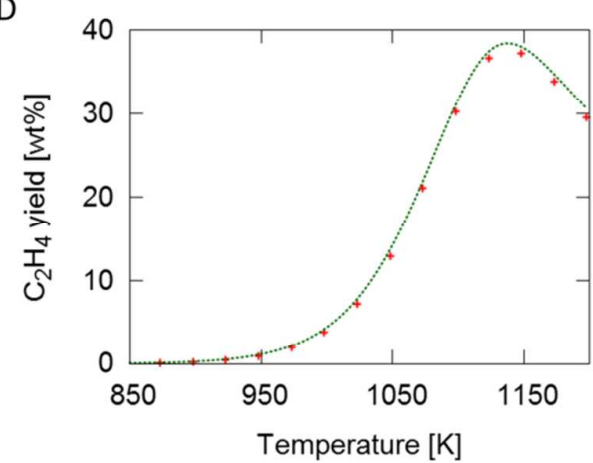

Figure S1: Conversion (A), hydrogen yield (B), -methane yield (C) and ethene yield (D) [wt\%] as a function of temperature $[\mathrm{K}]$ : +- full network, ......- reduced network.

Figure S2 shows the yields of propene and C4-(di)olefins. The maximum around $1075 \mathrm{~K}$ is slightly overpredicted by the reduced model but overall a satisfactory accuracy is obtained. 
A

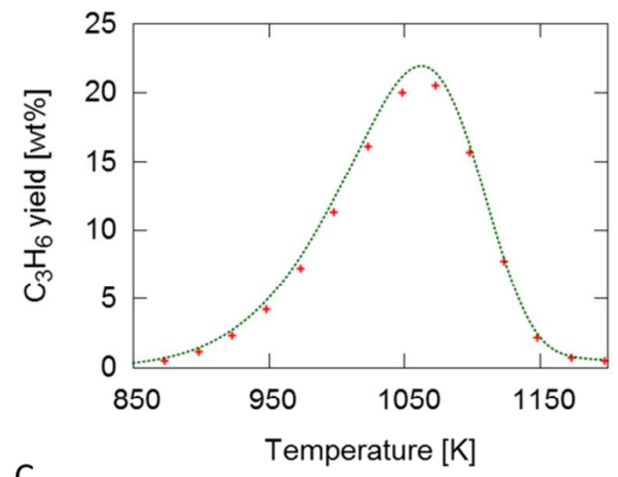

C

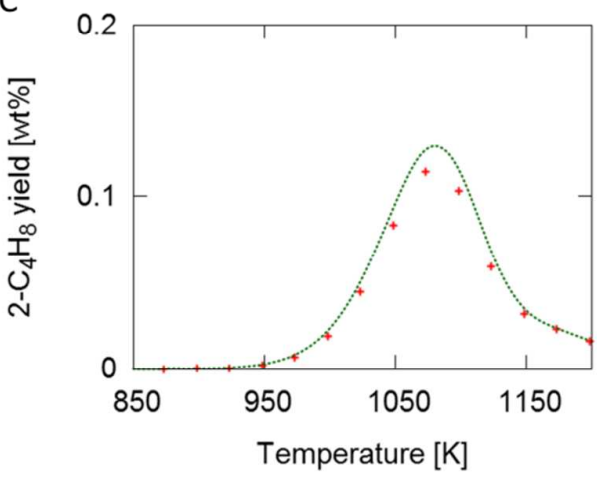

B

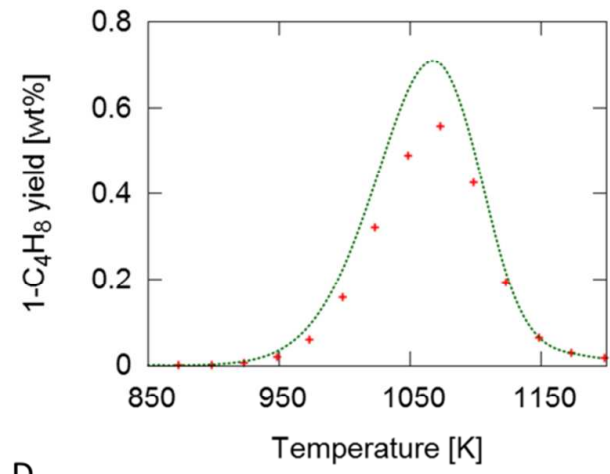

D

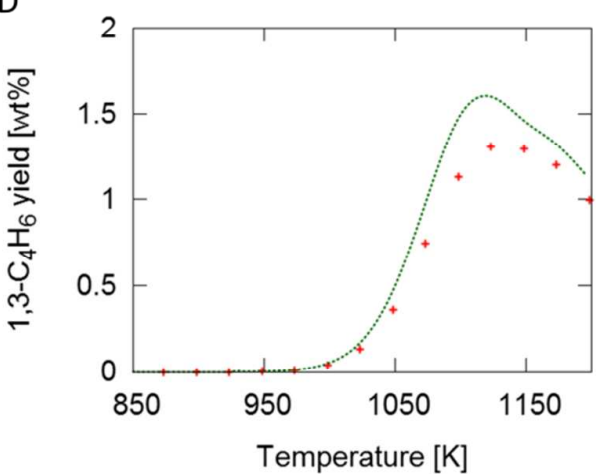

Figure S2: Propene (A), 1-butene yield (B), 2-butene yield (C) and 1,3-butadiene yield (D) [wt\%] as a function of temperature [K]: +- full network, .....- - reduced network.

Figure S3 shows the yields of 1,3-cyclopentadiene and the most abundant aromatic species, i.e. benzene, toluene and naphthalene. The agreement for 1,3-cyclopentadiene is good. Above $1150 \mathrm{~K}$, the yield of benzene is significantly underpredicted while the toluene and naphthalene yields are overpredicted. It is noted that these cracking severities is not reached in the performed 3D simulations. Indeed, the benzene, toluene and naphthalene yields in the simulated industrial propane cracker are around 2.4, 0.4 and $0.8 \mathrm{wt} \%$ respectively. The agreement between the reduced and the full network at these cracking severities is good. 


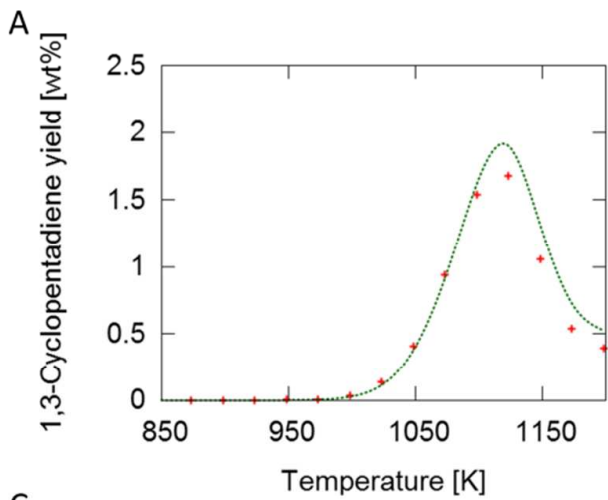

C

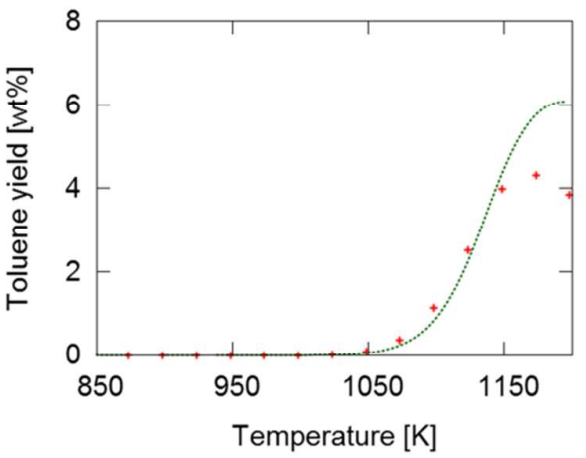

B

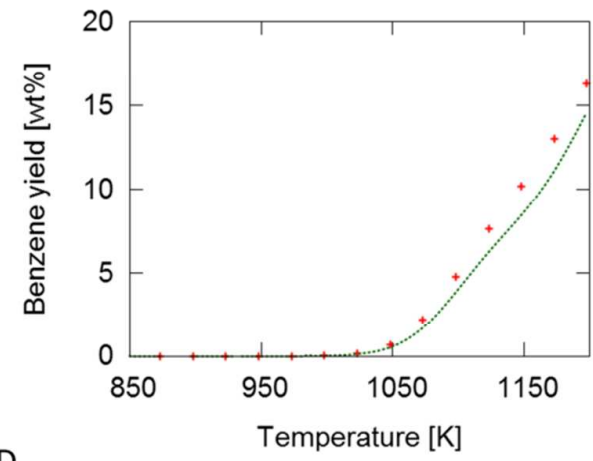

D

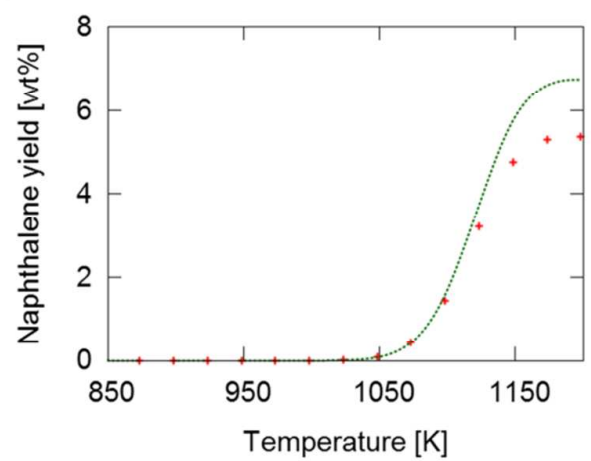

Figure S3: 1,3-cyclopentadiene (A), benzene yield (B), toluene yield (C) and naphthalene yield (D) [wt\%] as a function of temperature $[\mathrm{K}]:+-$ full network, ......- reduced network. 


\section{Industrial reactor simulation details}

The details of the Bare and SmallFins reactor are given in Table S1. The heat flux as a function of the reactor axial coordinate for the Bare reactor is shown in Figure S4. This heat flux profile is scaled for the SmallFins case to obtain the same total heat input as in the Bare case. Concretely, the heat flux profile of the bare reactor is multiplied with the ratio of the cross-sectional perimeters provided in Table S1, that is a scaling factor of $0.8243 \mathrm{~m} \mathrm{~m}^{-1}$.

The heat flux profile was obtained by performing a three-dimensional computational fluid dynamics simulation of the firebox with an industrially measured temperature profile as boundary condition on the reactor outer wall. In the present work, the coupling between the reactor side and firebox side has not been made. Although the present shape of the heat flux profile is realistic and industrially relevant, a higher accuracy on the heat flux profile could be obtained by performing a coupled simulation of the reactors and the firebox. However, this is beyond the scope of the present work as the main focus is to demonstrate the importance of three-dimensional simulations when assessing (enhanced) reactor geometries and to illustrate the implementation of the pseudo-steady state approximation in computational fluid dynamics. As the discussion of the present results focuses on the comparison between the Bare case and the SmallFins case, the absolute values of the yields for each case, possibly affected by small inaccuracies in the heat flux profile, are of secondary importance. The effects of the different reactor geometries relevant to each other are not affected by the shape of the heat flux profile.

Table S1: Details of industrial reactor geometries. Reproduced (Adapted) with permission from ref. ${ }^{2}$, Copyright 2013 American Institute of Chemical Engineers

\begin{tabular}{l|ll}
\hline Reactor ID & Bare & SmallFins \\
\hline Reactor length $[\mathrm{m}]$ & 10.556 & 10.556 \\
Adiabatic inlet section $[\mathrm{m}]$ & 0.444 & 0.444 \\
Maximum inner diameter $\left[10^{-3} \mathrm{~m}\right]$ & 30.2 & 31.3 \\
Number of fins $[-]$ & - & 24 \\
Helix angle $\left[{ }^{\circ}\right]$ & - & 33.1 \\
Outer diameter $\left[10^{-3} \mathrm{~m}\right]$ & 43.7 & 44.8 \\
Metal thickness $\left[10^{-3} \mathrm{~m}\right]$ & 6.75 & 6.75 \\
Fin height $\left[10^{-3} \mathrm{~m}\right]$ & - & 1.15 \\
Cross-sectional surface area $\left[10^{-6} \mathrm{~m}^{2}\right]$ & 715.7 & 715.7 \\
Cross-sectional perimeter $[\mathrm{m}]$ & 0.0948 & 0.1150 \\
\hline
\end{tabular}

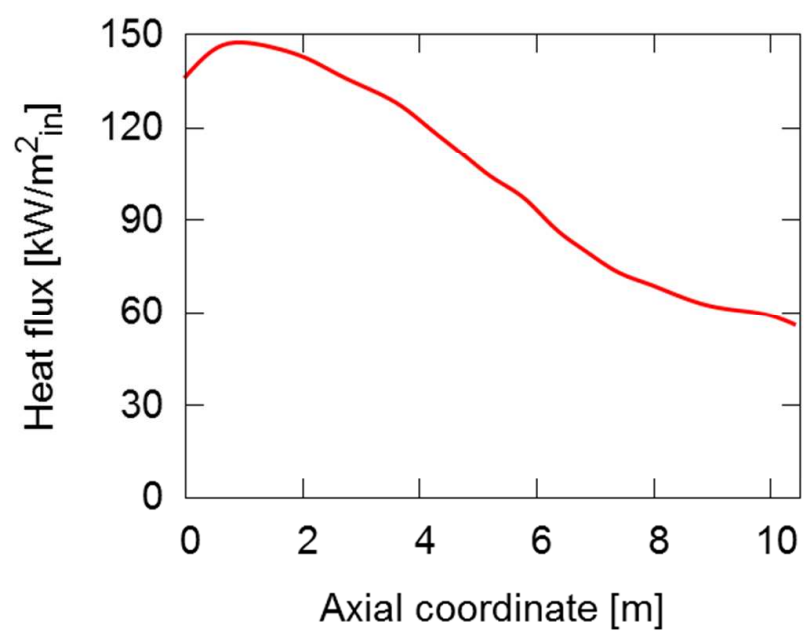

Figure S4: Heat flux $\left[\mathrm{kW} / \mathrm{m}^{2}{ }_{\mathrm{in}}\right]$ as a function of axial coordinate $[\mathrm{m}]$ for the Bare reactor case. 


\section{Averaging procedures}

In the following, all adopted procedures for averaging the three-dimensional data are discussed. Temperature is used as the averaged variable, but the procedures are similarly applied to other variables, e.g. pressure and species concentrations.

$\underline{\text { Mixing cup average over a cross-section as a function of axial position }}$

This procedure is illustrated in Figure S5. The mixing cup averaged temperature over a cross section $T_{m c}$ at axial position $z_{1}$ is defined as:

$$
T_{m c}=\frac{1}{\phi_{m, t o t}} \int_{0}^{r_{\max }} \int_{0}^{2 \pi} \phi_{m}\left(r, \theta, z_{1}\right) T\left(r, \theta, z_{1}\right) d r d \theta
$$

with $\phi_{m}$ the mass flow rate, $T$ the temperature, $r$ the radial coordinate, $\theta$ the azimuthal coordinate, $r_{\max }$ the maximum inner radius and $\phi_{m, t o t}$ the total mass flow rate $=$ $\int_{0}^{r_{\max }} \int_{0}^{2 \pi} \phi_{m}\left(r, \theta, z_{1}\right) d r d \theta$.

From the $3 \mathrm{D}$ results, this mixing cup averaged temperature is calculated by numerical integration over all cells $i$ of the cross section at an axial position $z_{1}$ :

$$
T_{m c}=\frac{1}{\phi_{m, t o t}} \sum_{i=1}^{\text {Ncells }} \phi_{m, i} T_{i}
$$

with $T_{i}$ the temperature of cell $\mathrm{i}, \phi_{m, i}$ the flow through cell $\mathrm{i}$, Ncells the number of cells in the cross-section and $\phi_{m, t o t}=\sum_{i=1}^{N c e l l s} \phi_{m, i}$.

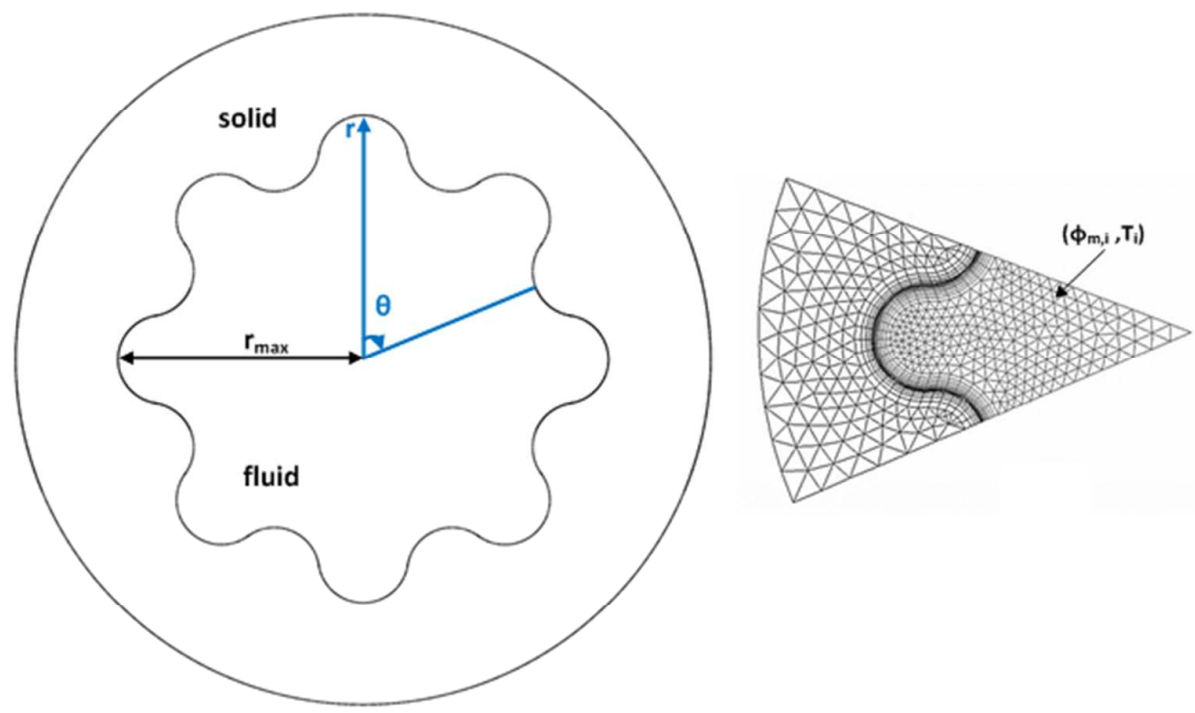

Figure S5: Illustration of the calculation of the mixing cup averaged temperature over a cross-section: a) entire cross section; b) grid of a cross section. Reproduced (Adapted) with permission from ref. 2, Copyright 2013 American Institute of Chemical Engineers 


\section{Azimuthally mixing cup average as function of radial position}

This procedure is illustrated in Figure S6. The mixing cup averaged temperature at a certain radial position $r_{1}$ at axial position $z_{1}$ is defined as:

$$
T_{a z}=\frac{1}{\phi_{m, r_{1}}} \int_{0}^{2 \pi} \phi_{m}\left(r_{1}, \theta, z_{1}\right) T\left(r_{1}, \theta, z_{1}\right) d \theta
$$

with $\phi_{m}$ the mass flow rate, $T$ the temperature, $\theta$ the azimuthal coordinate and $\phi_{m, t o t}$ the total mass flow rate at radial position $r_{1}=\int_{0}^{2 \pi} \phi_{m}\left(r_{1}, \theta, z_{1}\right) d \theta$.

From the simulation results, this mixing cup averaged temperature at a certain radial position $r_{1}$ at axial position $z_{1}$ is obtained by numerical integration over all cells $i$ of the cross section at axial position at axial position $z_{1}$ over a certain interval around the radial position $r_{1}$ :

$$
T_{\text {rad }}=\frac{1}{\phi_{m, t o t}} \sum_{i=1}^{N \text { cells }} \phi_{m, i} T_{i}, \forall i: r_{1}-\frac{\Delta r}{2}<r_{i}<r_{1}+\frac{\Delta r}{2}
$$

with $T_{i}$ the temperature of cell i, $\phi_{m, i}$ the flow through cell i, Ncells the number of cells in the cross-section, $\Delta r$ the interval width, $r_{i}$ radial coordinate of center of cell $\mathrm{i}$ and $\phi_{m, t o t}=$ $\sum_{i=1}^{N \text { cells }} \phi_{m, i}, \forall i: r_{1}-\frac{\Delta r}{2}<r_{i}<r_{1}+\frac{\Delta r}{2}$. In Figure 12 of the manuscript, the azimuthally mixing cup averaged values are plotted along the normalized radial position $r_{1, n o r m}$ calculated as $r_{1, \text { norm }}=r_{1} / r_{\max }$

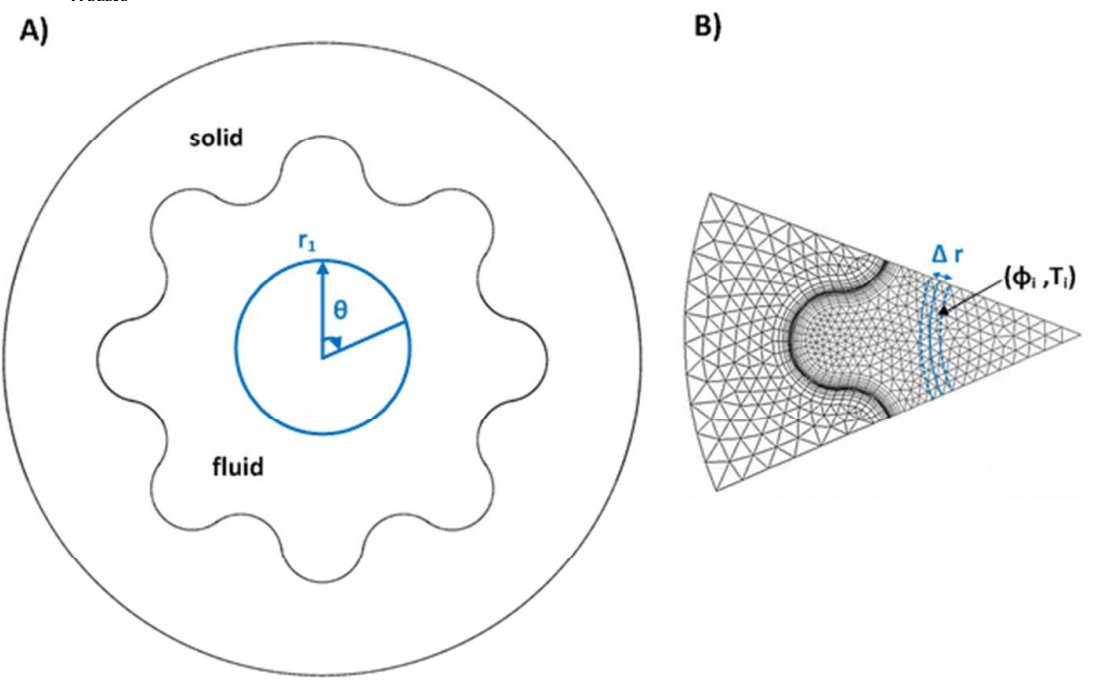

Figure S6: Illustration of calculation of the azimuthally mixing cup averaged temperature as a function of radial position: a) entire cross section; b) grid of a cross section. Reproduced (Adapted) with permission from ref. 2, Copyright 2013 American Institute of Chemical Engineers 


\section{Comparison of simulation results with reference data from industry}

Section 3.3 focuses on the differences seen in the CFD simulations of two different designs of an industrial reactor geometry. In this discussion, the section downstream of the reactor is considered to have little impact on the yield differences observed. For direct comparison with the industrially measured data however, it is important to include the transfer line heat exchanger (TLE), where the process gas is quenched to halt any potential secondary reactions.

The quenching zone of the present Millisecond furnace type, consists of an adiabatic section with a length of $0.82 \mathrm{~m}$, where the effluent of four reactors is joined. This is followed by a $11.68 \mathrm{~m}$ TLE with an internal diameter of $66.6 \mathrm{~mm}$. External to this tube, water at its saturation temperature is used to generate 140 bar steam with a 5\% quality. At the exit of the TLE, the process gas is quenched to a temperature of $457{ }^{\circ} \mathrm{C}$ at which point no significant reactions are taking place anymore.

Table S2 shows the results obtained when including this quenching sections as a $1 \mathrm{D}$ plug flow heat exchanger at the outlet of the 3D simulations. For the present operating conditions, an additional $10 \%$ conversion is achieved in the TLE. Comparing the yield differences between the Bare and SmallFins cases before and after the TLE indicates that the relative differences are maintained and the inclusion of the TLE is not strictly necessary in order to draw conclusions on the reactor performance of different geometries. The combined model however does allow a better comparison with the industrial data. Relatively good agreement is seen taking into account the accuracy of industrial yield measurements (5 rel.\%), the uncertainty on the feedstock composition and the slightly different operating conditions.

Table S2: Comparison of simulated product yields with industrial data. Reactor simulation done via computational fluid dynamics, TLE simulation done via 1D calculation.

\begin{tabular}{l|rr|rr|r}
\hline & Bare_3D & SmallFins_3D & Bare_3D+TLE & SmallFins_3D+TLE & Industrial \\
\hline $\mathrm{H}_{2}$ & 2.03 & 2.01 & 2.27 & 2.26 & 1.58 \\
$\mathrm{CH}_{4}$ & 14.67 & 14.77 & 18.96 & 19.05 & 19.31 \\
$\mathrm{C}_{2} \mathrm{H}_{2}$ & 0.66 & 0.65 & 0.66 & 0.65 & 0.76 \\
$\mathrm{C}_{2} \mathrm{H}_{4}$ & 26.72 & 26.57 & 32.97 & 32.82 & 35.15 \\
$\mathrm{C}_{2} \mathrm{H}_{6}$ & 1.15 & 1.13 & 1.92 & 1.90 & 2.85 \\
$\mathrm{C}_{3} \mathrm{H}_{4}$ & 0.78 & 0.77 & 0.64 & 0.64 & 0.71 \\
$\mathrm{C}_{3} \mathrm{H}_{6}$ & 19.46 & 19.57 & 15.45 & 15.53 & 16.98 \\
$\mathrm{C}_{3} \mathrm{H}_{8}$ & 25.81 & 25.80 & 15.82 & 15.84 & 15.67 \\
$13-\mathrm{C}_{4} \mathrm{H}_{6}$ & 1.42 & 1.41 & 1.77 & 1.77 & 1.88 \\
$1-\mathrm{C}_{4} \mathrm{H}_{8}$ & 0.95 & 0.95 & 0.58 & 0.59 & 1.24 \\
$2-\mathrm{C}_{4} \mathrm{H}_{8}$ & 0.13 & 0.13 & 0.12 & 0.12 & \\
$\mathrm{i}-\mathrm{C}_{4} \mathrm{H}_{8}$ & 0.06 & 0.06 & 0.07 & 0.07 & 0.22 \\
$\mathrm{n}-\mathrm{C}_{4} \mathrm{H}_{10}$ & 0.01 & 0.01 & 0.02 & 0.02 & 0.13 \\
$13-\mathrm{CPD}$ & 0.19 & 0.19 & 1.62 & 1.61 & 0.34 \\
Me-13-CPD & 0.36 & 0.36 & 0.34 & 3.98 & \\
Benzene & 2.32 & 2.34 & 3.96 & 0.77 & \\
Toluene & 0.37 & 0.36 & 0.78 & 0.16 & \\
Styrene & 0.09 & 0.09 & 0.16 & 1.17 & \\
Naphthalene & 0.78 & 0.77 & 1.18 & & \\
\hline
\end{tabular}

CPD: cyclopentadiene 


\section{Speedup of 3D simulations by implementing PSSA}

The speedup factors in Table 4 were computed by running two simulations for each feedstock: in one simulation the pseudo-steady state assumption was applied to the kinetic network while in the other simulation the kinetic network was directly solved. The simulations were initialized based on the boundary conditions of the inlet and subsequently allowed to iterate until the relative change in the species mass fractions in the outlet between the last iteration and (last iteration - 200) is smaller than $10^{-4}$. The speedup factor is calculated as the ratio of the total required CPU-time for the non-PSSA simulation and the PSSA simulation. All simulations were run on a compute node equipped with two 4-core Intel $\mathbb{R}$ Xeon ${ }^{\circledR}$ L5520 CPUs (Nehalem architecture, 2.27 Ghz, 8 MB L3 cache), 12 GB DDR3 RAM at $1067 \mathrm{MHz}$ and $146 \mathrm{~GB}$ local disk space on a 10k SAS disk.

The speedup factors reported in the manuscript (Table 4) include two effects: the speedup due to the chemistry reduction and the speedup due to the more favorable numerical settings. The simulation without PSSA and with PSSA were run independently so with the most favorable numerical settings for each simulation. Within each simulation, the under-relaxation factor has been kept the same for all the species. The direct speedup effect of the pseudo-steady state assumption originates from the reduction in the number of species transport equations that are to be solved and the reduction in the stiffness of the system. However the elimination of the species with the shortest life times from the Jacobian matrix favors the stability of the matrix inversion algorithm, allowing higher under-relaxation factors to be used in the PSSA case. This secondary effect is a direct consequence of the implementation of PSSA. According to this reasoning, the speedup due to the numerical settings can also be attributed to the implementation of PSSA and can hence be included in the overall speedup for this method.

Note that using an under-relaxation factor of 0.001 on the species was necessary in the first iterations with the species equations enabled. In subsequent iterations, the under-relaxation factors were increased to more reasonable values between 0.2 and 0.9 , depending on the stability of the simulation. In the simulations without PSSA, the under-relaxation factors are generally lower compared to the simulations with PSSA. In general, the larger the spread on the lifetime of the species, the lower the under-relaxation of the simulation should be, to preserve stability.

The speedup simulations have been carried out in a second way in order to try to abstract the effect of under-relaxation. In this case, the numerical settings for the PSSA case and the nonPSSA case have been kept identical for each feedstock, using for both simulations the underrelaxation factor of the more unstable non-PSSA case. The simulations have been run until the residuals on all the variables were below $10^{-8}$. The speedup factor calculated via this method isolates the effect of the reduction in the number of species and the lower computational cost of determining the rate of formation of the species. All simulations were run on a compute node equipped with two 8-core Intel ${ }^{\circledR}$ Xeon ${ }^{\circledR}$ E5-2670 CPUs (Sandy Bridge architecture, 2.60 Ghz, $20 \mathrm{MB}$ L3 cache), 64 GB DDR3 RAM at $1600 \mathrm{MHz}, 500 \mathrm{~GB}$ local disk space on a 7.2k SATA disk and 400 TB shared memory on 7.2k SAS disks connected to the compute nodes via a fat tree topology FDR Infiniband network with a peak bandwidth of 9.5 GB s-1. The results of the new simulations are provided in Table S3. 
Table S3: Specifications of the reaction models and the obtained speedup factor in the 3D validation simulations with consistent numerical setup between PSSA case and non-PSSA case.

\begin{tabular}{l|ccc|c}
\hline Feedstock & Nr molecules & Nr radicals & Nr reactions & Speedup \\
\hline Ethane & 6 & 3 & 9 & 2.6 \\
Butane & 8 & 6 & 27 & 6.6 \\
Propane & 13 & 11 & 100 & 13.2 \\
\hline
\end{tabular}

The simulations with identical numerical settings display a lower speedup factor, indicating the significant importance of the contribution of numerical settings in speeding up a simulation. However even without the contribution of numerical settings, speedup factors up to 13.2 can be reached for a system with 11 radicals, 13 molecules and 100 reactions. 


\section{Verification of the stiffness reduction as a consequence of applying PSSA}

\subsection{Stiffness of kinetic equations}

Kinetic models show a large spread in chemical time scales associated with species and reactions. Stiffness is therefore an important feature of chemical kinetic differential equations and any method which decreases the stiffness of the kinetic equations may result in considerable savings of computer time.

The kinetics in a plug flow reactor can be described by the following system of differential equations

$$
\frac{\partial\left(c_{i}\right)}{\partial t}+\nabla \cdot\left(c_{i} \boldsymbol{u}\right)=\nabla \cdot\left(D_{i} \nabla c_{i}\right)+R_{i}
$$

Where $c_{i}$ is the concentration of species $i, R_{i}$ is the production rate of species $i, D_{i}$ is the diffusion coefficient of species $i$ and $\boldsymbol{u}$ is the flow velocity. The stiffness of this system of differential equations can be characterized by the stiffness ratio, which depends on the eigenvalues $\lambda$ of the chemical Jacobian $\boldsymbol{J}^{3}$. The chemical Jacobian is given by

$$
\boldsymbol{J}=\left(J_{i j}\right)=\frac{\partial R_{i}(\boldsymbol{c})}{\partial c_{j}}
$$

where $\boldsymbol{c}$ is the n-dimensional concentration vector. The stiffness ratio is given by

$$
\text { stiffness ratio }=\frac{\max \left|\Re\left(\lambda_{i}\right)\right|}{\min \left|\Re\left(\lambda_{j}\right)\right|}
$$

where $\Re\left(\lambda_{i}\right)$ denotes the real part of eigenvalue $\lambda_{i}$ of the Jacobian $\boldsymbol{J}$. The stiffness ratio is the ratio of the fastest transient in the system, i.e. the largest $\mathfrak{R}\left(\lambda_{i}\right)$, to the slowest transient, i.e. the smallest $\Re\left(\lambda_{i}\right)$. Stiff systems are characterized by a stiffness ratio much larger than 1 .

One way of reducing the stiffness of kinetic models is to remove the fastest transients from the system of differential equations. This is possible by applying the pseudo-steady state assumption to the highly reactive species.

\subsection{Species lifetime and the PSSA}

The species involved in chemical mechanisms each have a characteristic chemical lifetime. For a molecule of species $i$, chemical lifetime is defined as the time elapsed from its creation to its consumption. Species with the shortest overall lifetimes are usually highly reactive (radical) species and could potentially be treated as PSSA species ${ }^{4}$. A generalized interpretation of the species lifetime is based on the diagonal elements of the chemical Jacobian $^{5}$.

$$
\tau_{i}=-\frac{1}{J_{i i}}
$$


It can be shown ${ }^{4,5}$ that the instantaneous error induced on a single species by applying the PSSA to that species is given by

$$
\Delta c_{i}^{s}=\frac{1}{J_{i i}} R_{i}
$$

The PSSA hence relates to the diagonal elements of the Jacobian, while stiffness was related to its eigenvalues. A relation between the eigenvalues and diagonal elements is therefore required in order to relate stiffness to the PSSA.

\subsection{Jacobian properties for steam cracking of propane}

In order to evaluate the properties of the chemical Jacobian, the pyrolysis of propane was simulated with the one-dimensional plug flow model implemented in CHEMKIN. An isothermal simulation $(\mathrm{T}=1073.15 \mathrm{~K})$ was performed on the Millisecond reactor geometry using the complete propane network of 85 species. The propane mass flow rate, steam dilution and pressure were set to $43.66 \cdot 10^{-3} \mathrm{~kg} \mathrm{~s}^{-1}, 0.325 \mathrm{~kg} \mathrm{~kg}^{-1}$ and $2 \mathrm{~atm}$ respectively. The reactor inner diameter was $0.0302 \mathrm{~m}$. The PSSA was not applied to any species.

Subsequently, KINALC ${ }^{5}$ was used to calculate the Jacobian elements and eigenvalues, based on temperature, pressure and concentrations at the outlet of the reactor. The results are shown in Table S4. In this table, a large difference in lifetime between the molecular and the radical species can be observed. The radical species correspond to large diagonal elements and for each of these large diagonal elements a comparable eigenvalue can be found. Removing the radical species with large diagonal elements $J_{i i}$, or equivalently with a small lifetime, therefore reduces the stiffness of the system.

The relation between eigenvalues and diagonal elements also has a mathematical funding, as is shown below.

\subsection{Relating eigenvalues and diagonal elements}

Coincidence of eigenvalues and diagonal elements is good in case of nearly diagonal or nearly triangular matrices ${ }^{6}$. The chemical Jacobian is in many cases nearly triangular. This characteristic can be seen more clearly when the rows and columns of the matrix are ordered according to the value of the diagonal elements. In Figure S7 the Jacobian elements are shown for the pyrolysis of propane at the conditions described above. The elements in the lower triangular matrix are markedly less significant than those in the upper triangular matrix.

For a triangular matrix, the eigenvalues are exactly equal to the diagonal elements. For nearly triangular matrices, the diagonal elements serve as an approximation for the eigenvalues. Assume that $\boldsymbol{A}$ is an upper triangular matrix and $\boldsymbol{E}$ a lower triangular matrix with small elements. Using Wilkinson's perturbation theory an upper bound on the deviation of the eigenvalues of $\boldsymbol{A}+\boldsymbol{E}$ from those of $\boldsymbol{A}$ is given by $\|\boldsymbol{E}\| / s(\lambda)$. Herein, $s(\lambda)$ is the condition number of the eigenvalue $\lambda^{5-9}$.

$$
s(\lambda)=\left\|\boldsymbol{y}^{\boldsymbol{T}} \boldsymbol{x}\right\|_{2}
$$


where $\boldsymbol{y}^{\boldsymbol{T}}$ and $\boldsymbol{x}$ are the normalized left and right eigenvector associated with $\lambda$, respectively. In practice, a small condition number $s(\lambda)$ is only expected if two eigenvalues have almost the same value ${ }^{10}$. The deviation will therefore be much larger for the small diagonal elements where the lower matrix coefficients are still significant and the differences between the absolute values of the eigenvalues are smaller. However, the opposite holds for the large diagonal elements: large diagonal elements always correspond to large eigenvalues, as is also shown in Table S4. Removing species with large diagonal elements $J_{i i}$, or equivalently with a small lifetime, therefore reduces the stiffness of the system, which is done here.

Table S4. Comparison of the eigenvalues with the diagonal Jacobian elements for selected species of the propane network at the outlet of the isothermal millisecond reactor $(T=1073.15 \mathrm{~K})$.

\begin{tabular}{l|lll}
\hline Species $\boldsymbol{i}$ & Lifetime $\boldsymbol{\tau}_{\boldsymbol{i}}$ & Diagonal $\boldsymbol{J}_{\boldsymbol{i} \boldsymbol{i}}$ & Eigenvalue $\boldsymbol{\lambda}_{\boldsymbol{i}}$ \\
\hline $\mathrm{H}$. & $8.80 \mathrm{E}-09$ & $-1.137 \mathrm{E}+08$ & $-1.158 \mathrm{E}+08$ \\
IC4H9. & $9.70 \mathrm{E}-09$ & $-1.031 \mathrm{E}+08$ & $-1.115 \mathrm{E}+08$ \\
2-C4H9. & $1.29 \mathrm{E}-08$ & $-7.735 \mathrm{E}+07$ & $-7.737 \mathrm{E}+07$ \\
1-C3H7. & $2.04 \mathrm{E}-08$ & $-4.897 \mathrm{E}+07$ & $-4.873 \mathrm{E}+07$ \\
1-C4H9. & $3.14 \mathrm{E}-08$ & $-3.189 \mathrm{E}+07$ & $-3.190 \mathrm{E}+07$ \\
1-C4-4. & $2.02 \mathrm{E}-07$ & $-4.964 \mathrm{E}+06$ & $-5.517 \mathrm{E}+06$ \\
C2H3. & $2.99 \mathrm{E}-07$ & $-3.346 \mathrm{E}+06$ & $-3.391 \mathrm{E}+06$ \\
2-C3H7. & $3.79 \mathrm{E}-07$ & $-2.637 \mathrm{E}+06$ & $-1.974 \mathrm{E}+06$ \\
C2H5. & $6.87 \mathrm{E}-07$ & $-1.456 \mathrm{E}+06$ & $-8.204 \mathrm{E}+05$ \\
CH3. & $2.27 \mathrm{E}-06$ & $-4.429 \mathrm{E}+05$ & $-2.724 \mathrm{E}+05$ \\
1-C4-3. & $2.63 \mathrm{E}-05$ & $-3.807 \mathrm{E}+04$ & $-3.979 \mathrm{E}+04$ \\
C3H5. & $3.85 \mathrm{E}-05$ & $-4.290 \mathrm{E}+04$ & $-3.120 \mathrm{E}+04$ \\
1,3-C4H6 & $2.09 \mathrm{E}-02$ & $-4.791 \mathrm{E}+01$ & $-2.763 \mathrm{E}+01$ \\
C2H2 & $2.72 \mathrm{E}-02$ & $-3.683 \mathrm{E}+01$ & $-2.494 \mathrm{E}+01$ \\
N1C4H8 & $3.85 \mathrm{E}-02$ & $-2.595 \mathrm{E}+01$ & $-1.787 \mathrm{E}+01$ \\
C3H4(PD) & $4.01 \mathrm{E}-02$ & $-2.492 \mathrm{E}+01$ & $-1.283 \mathrm{E}+01$ \\
C2H4 & $4.83 \mathrm{E}-02$ & $-2.069 \mathrm{E}+01$ & $-5.298 \mathrm{E}+00$ \\
C3H6 & $5.86 \mathrm{E}-02$ & $-1.699 \mathrm{E}+01$ & $-4.683 \mathrm{E}+00$ \\
N2C4H8 & $6.94 \mathrm{E}-02$ & $-1.442 \mathrm{E}+01$ & $-4.237 \mathrm{E}+00$ \\
NC4H10 & $2.13 \mathrm{E}-01$ & $-4.688 \mathrm{E}+00$ & $-1.948 \mathrm{E}+00$ \\
C3H8 & $3.89 \mathrm{E}-01$ & $-2.562 \mathrm{E}+00$ & $-1.582 \mathrm{E}+00$ \\
C2H6 & $6.59 \mathrm{E}-01$ & $-1.517 \mathrm{E}+00$ & $-1.104 \mathrm{E}+00$ \\
H2 & $7.14 \mathrm{E}-01$ & $-1.401 \mathrm{E}+00$ & $-7.027 \mathrm{E}-01$ \\
CH4 & $5.82 \mathrm{E}+00$ & $-1.719 \mathrm{E}-01$ & $-6.394 \mathrm{E}-06$ \\
\hline
\end{tabular}




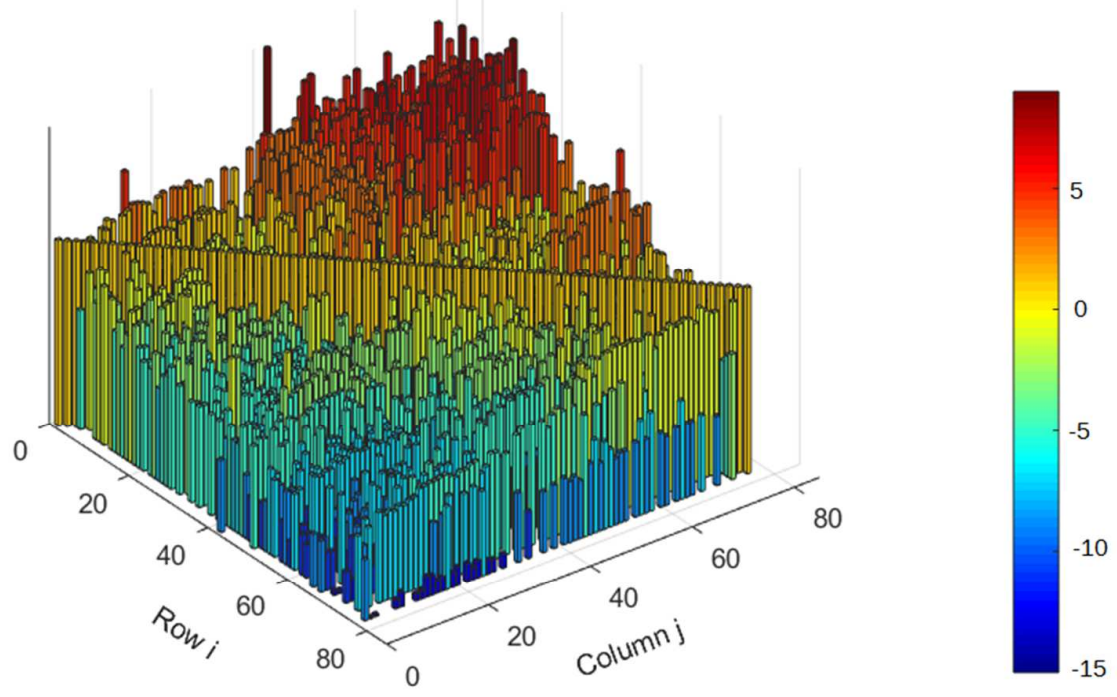

Figure S7. Illustration of nearly triangular Jacobian for the propane network at the outlet of the ISOTHERMAL millisecond reactor $(t=1073.15 \mathrm{~K})$. The height of the columns represents the $\log$ of the absolute value of the Jacobian element relative to the diagonal element in the same row. The species are ordered in terms of decreasing lifetime. 


\section{One-dimensional simulations implementing PSSA}

\subsection{Simulation setup}

COILSIM1D is a $1 \mathrm{D}$ reactor model for steam cracking reactors incorporating a single-event microkinetic model of more than 700 species ${ }^{11}$. The pseudo-steady state approximation is applied both a priori and on-the-fly to reduce the computational cost of such an extensive kinetic model. The governing equations of COILSIM1D are:

$$
\begin{gathered}
\frac{d F_{j}}{d z}=\frac{\pi d^{2}}{4} R_{a v g, j} \\
-\frac{d p}{d z}=\left(\frac{2 f}{d}+\frac{\zeta}{\pi r_{b}}\right) \rho u^{2}+\rho u \frac{d u}{d z} \\
\sum_{j=1}^{N_{s}} F_{j} c_{p_{j}} \frac{d T_{a v g}}{d z}=\pi d_{i n} q_{i n}+\frac{\pi d_{i n}^{2}}{4} \sum_{i=1}^{N_{R}} r_{i}\left(-\Delta H_{i}\right)
\end{gathered}
$$

with $f=\alpha_{f} f_{\text {corr }}, f_{\text {corr }}$ calculated from the Colebrook equation ${ }^{12}$ and $\alpha_{f}$ a user-supplied correction factor to account for the increased pressure drop by application of a threedimensional reactor technology. For the simulations, $\alpha_{f}$ and $\alpha_{h}$ were set to unity for the Bare tube, while for the SmallFins tube, $\alpha_{f}$ and $\alpha_{h}$ were set to 1.98 and 1.52 respectively. These values were obtained from the non-reactive flow simulations of our previous work ${ }^{2}$.

COILSIM1D was extended to account for the influence of the radial temperature and $\beta$ radical concentration profiles on product yields and heat transfer by calculating the mixing cup rate of formation $R_{a v g, j}$ in equation (S4) over the cross-sectional area at each axial grid point $z_{i}$ during numerical integration of the ordinary differential equations (S4), (S11) and (S12). The well-known power law velocity profile for fully developed pipe flow proposed by Prandtl ${ }^{13}$ is assumed:

$$
u(r)=u_{\max }\left(1-\frac{2 r}{d_{\text {in }}}\right)^{1 / n}
$$

where $\mathrm{n}$ is typically taken to be 7 resulting in the one-seventh law. From heat-momentum similarity, the following radial temperature distribution is assumed:

$$
T(r)=T_{\text {int }}+\left(T_{\text {center }}-T_{\text {int }}\right)\left(1-\frac{2 r}{d_{\text {in }}}\right)^{1 / n}
$$

Figure S8 shows a flow sheet of the adopted calculation procedure. At the first point of the axial grid, i.e. $\mathrm{i}=0$ in Figure $\mathrm{S} 8$, the average temperature $T_{\text {avg }}$ is the reactor inlet temperature supplied by the user and the inner wall temperature is calculated using the heat flux at this axial position:

$$
q_{\text {in }}=h\left(T_{i n t}-T_{a v g}\right)
$$


with $q_{\text {in }}$ the heat flux per inner wall surface area, $h=\alpha_{h} h_{\text {corr }}, \alpha_{h}$ a user-supplied correction factor and $h_{\text {corr }}$ calculated from the Gnielinski correlation ${ }^{14}$ :

$$
N u=\frac{\frac{f}{8}(R e-1000) \operatorname{Pr}}{1+12.7\left(\frac{f}{8}\right)^{1 / 2}\left(\operatorname{Pr}^{2 / 3}-1\right)}
$$

Next, $T_{\text {center }}$ is calculated by calculating the mixing cup temperature from equation (S15) and setting it equal to the value of the reactor inlet temperature:

$$
T_{a v g}=\frac{\int_{0}^{A} \dot{m}(r) T(r) d A}{\int_{0}^{A} \dot{m}(r) d A}
$$

Hence, the radial temperature profile of equation (S15) is defined. From this profile, the mixing cup rates of formation $R_{a v g, j}$ are calculated:

$$
R_{a v g, j}=\frac{\int_{0}^{\frac{d_{i n}}{2}} \rho(r) u(r) R_{j}(\boldsymbol{c}(r), T(r)) d r}{\int_{0}^{\frac{d_{\text {in }}}{2}} \rho(r) u(r) d r}
$$

By substitution of the ideal gas law and neglecting the radial variation of pressure and molecular mass, equation (S19) becomes:

$$
R_{a v g, j}=\frac{\int_{0}^{\frac{d_{i n}}{2}} \frac{u(r) R_{j}(\boldsymbol{c}(r), T(r))}{T(r)} d r}{\int_{0}^{\frac{d_{i n}}{2}} \frac{u(r)}{T(r)} d r}
$$

With this $R_{a v g, j}$, the plug flow equations (S4), (S11) and (S12) are integrated over $\Delta \mathrm{z}_{i}$, the axial length of the axial grid point $\mathrm{z}_{\mathrm{i}}$. Hence, updated values for $\boldsymbol{c}$ and $T_{\text {avg }}$ are available. From equation (S16), $T_{\text {int }}$ is updated. If the difference between the old and the updated value of $T_{\text {avg }}$ is smaller than the threshold value $\varepsilon_{\mathrm{T}}$, set to $0.01 \%$, convergence is reached in this axial grid point and the simulation is proceeded to the next axial grid point. The converged $T_{a v g}$ and $T_{i n t}$ are used as initial guess for the next grid point. This procedure is repeated until the end of the reactor is reached, i.e. $z_{i}=L$. 


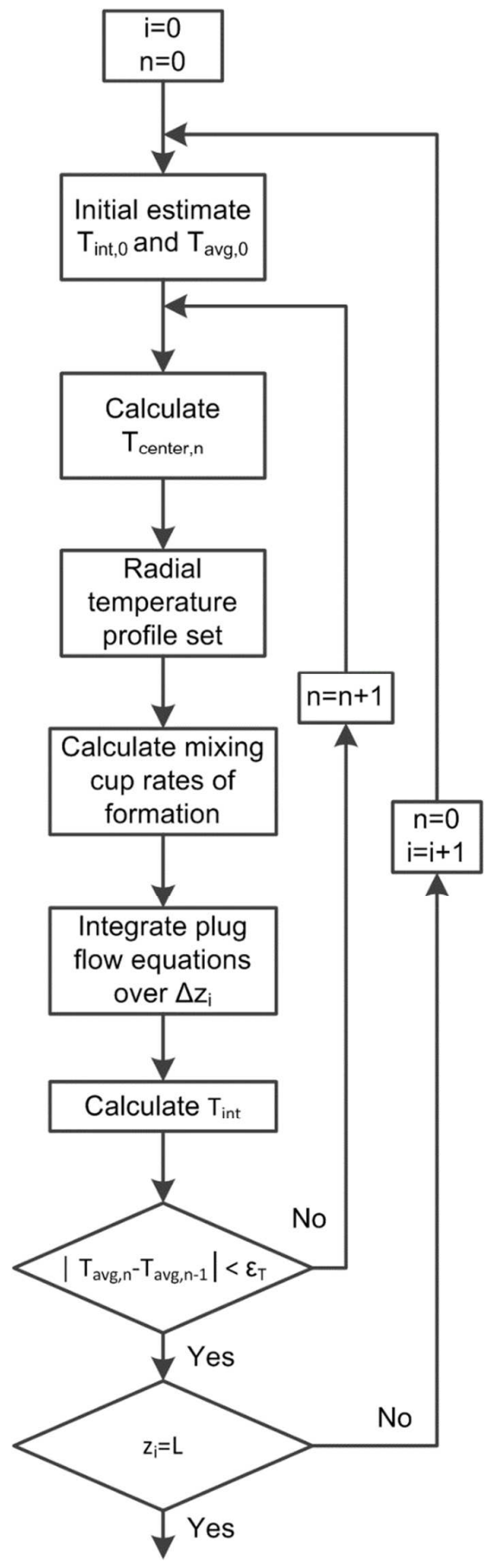

Figure S8: Flow sheet of calculation procedure in COILSIM1D to account for radial profiles.

Three types of simulations were performed. First without taking into account any radial variation, referred to as ' $1 \mathrm{D}$ '. Second, taking into account the radial temperature profile, referred to as ' $1 \mathrm{D} \mathrm{T}$ ', without accounting for radial variation in the species concentrations: 


$$
R_{a v g, j}=\frac{\int_{0}^{\frac{d_{i n}}{2}} \frac{u(r) R_{j}(c, T(r))}{T(r)} d r}{\int_{0}^{\frac{d_{i n}}{2}} \frac{u(r)}{T(r)} d r}
$$

Finally, by taking into account both the radial temperature profile and the radial $\beta$-radials concentration profile. The radial variation of the concentration of the molecules is neglected in all simulations as it has been shown to be much smaller than the radial variation of the $\beta$ radicals concentration ${ }^{2,15}$. The $\beta$-radicals concentrations as a function of radial coordinate are obtained by solving the algebraic equations resulting from application of the PSSA to the $\beta$ radicals as a function of the radial coordinate:

$$
-R_{\beta_{j}}^{c}\left(\boldsymbol{c}_{\boldsymbol{M}}, \boldsymbol{c}_{\boldsymbol{\beta}}(r), T(r)\right)=R_{\beta_{j}}^{f}\left(\boldsymbol{c}_{\boldsymbol{M}}, \boldsymbol{c}_{\boldsymbol{\beta}}(r), T(r)\right)
$$

Hence, equation (S19) becomes:

$$
R_{a v g, j}=\frac{\int_{0}^{\frac{d_{i n}}{2}} \frac{u(r) R_{j}\left(\boldsymbol{c}_{\boldsymbol{M}}, \boldsymbol{c}_{\boldsymbol{\beta}}(r), T(r)\right)}{T(r)} d r}{\int_{0}^{\frac{d_{i n}}{2}} \frac{u(r)}{T(r)} d r}
$$

\subsection{Results and discussion}

To assess the error when using a one-dimensional plug flow model, simulations were performed using COILSIM1D. Although COILSIM1D normally comes with an extensive single-event microkinetic model containing about 700 species and thousands of reactions, the microkinetic model adopted in the 3D simulations was used in these COILSIM1D simulations. Furthermore, the algorithm of application of PSSA on the $\beta$-radicals, the thermodynamic data of the species and the reactor alloy properties of the $3 \mathrm{D}$ simulations were implemented to isolate the effect of the adopted reactor model on the simulation results.

Table S5 compares the 1D simulation results with the 3D simulation results of the two reactors discussed in the paper. For the two reactor designs, three 1D simulations were performed; without radial profiles (1D), with a radial temperature profile (1D T) and with a radial temperature and $\beta$-radicals profile (1D T, $\beta$ ). In all 1D simulations, the heat flux profile to the reactors was scaled to obtain the same propane conversion as in the corresponding $3 \mathrm{D}$ simulation. The COT in the 1D simulations is higher than in the 3D simulations. Taking into account radial profiles results in a closer agreement between the 1D and 3D simulated COT's. The pressure drop in the Bare reactor is slightly overpredicted in the 1D model compared to the 3D results. For the SmallFins reactor, a good agreement is obtained. This shows that using a correction factor obtained from non-reactive CFD simulations can give accurate pressure drop predictions for reactive 1D simulations. However, it should be noted that the Reynolds number of the non-reactive simulation and this reactor simulation is the same which is obviously important to obtain a correct scaling factor.

Figure S9 A and B compare the external tube wall temperature (TMT) in the 3D and the 1D simulations for the Bare and the SmallFins reactor respectively. For the Bare reactor, the maximum tube metal temperature is overpredicted by $26 \mathrm{~K}$ using the $1 \mathrm{D}$ model without radial 
profiles. As shown by Sundaram and Froment ${ }^{16}$, the temperature difference between the inner wall temperature and the mixing cup process gas temperature is indeed larger when using a correlation for the Nusselt number derived for non-reactive flow than when accounting for the endothermic reactions. Consequently, a good agreement is seen between 1D and 3D TMT's towards the end of the reactor where the endothermic heat of reaction is lower. Furthermore, axial conduction in the tube metal is not accounted for in the 1D model, which flattens out the TMT profile in the 3D simulations, reducing the TMT maximum. Taking into account the effect of only the radial temperature and also $\beta$-radicals concentration profile reduces the overprediction of the maximum TMT to 20 and $13 \mathrm{~K}$ respectively. Similar considerations as made for the Bare reactor can be made for the SmallFins reactor, with a maximum TMT difference compared to the 3D simulation equal to 20,18 and $14 \mathrm{~K}$ for $1 \mathrm{D}, 1 \mathrm{D} \mathrm{T}$ and $1 \mathrm{D} \mathrm{T}, \beta$ respectively.
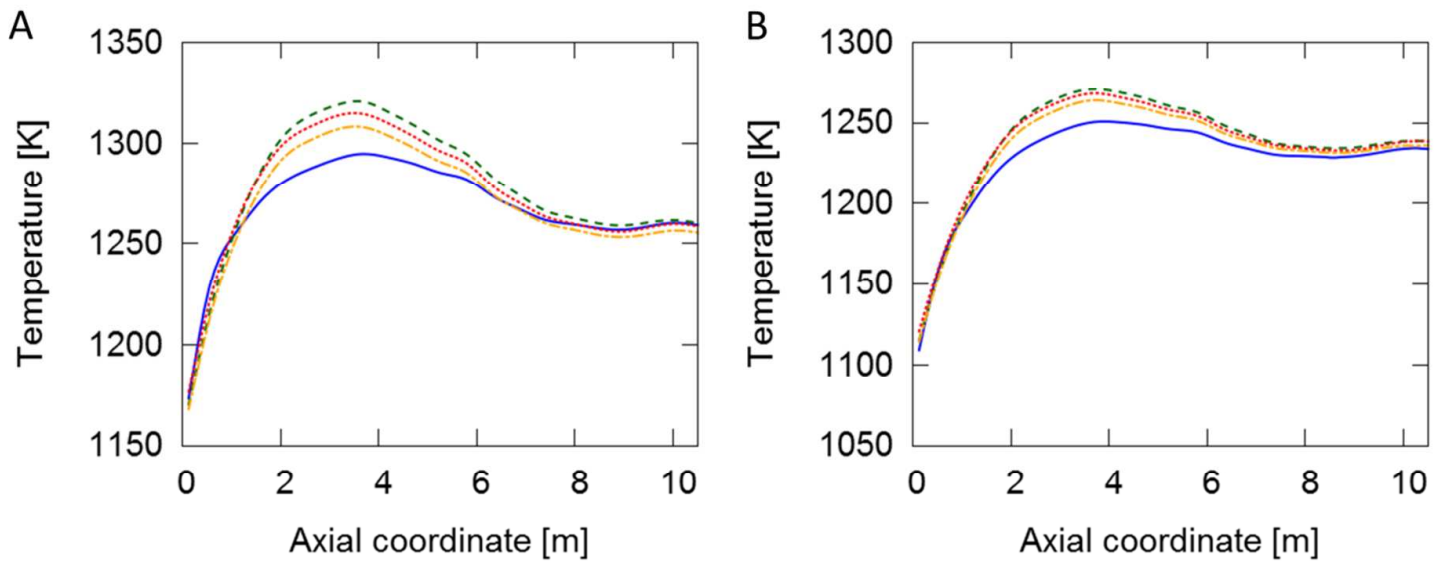

Figure S9: External tube metal temperature $[\mathrm{K}]$ for the Bare (A) and SmallFins (B) reactor: - - 3D; - - - 1D; ....... - 1D T; --.- - 1D T, $\beta$.

Figure S10 shows the propane conversion for the three-dimensional and the one-dimensional simulations of the Bare reactor. Without film cracking, the induction length for reaction to start is longer compared to the 3D simulation. When accounting for film cracking in the 1D simulations, the agreement with the $3 \mathrm{D}$ conversion profile at the start of the reactor is better as seen from the insert in Figure S10. 


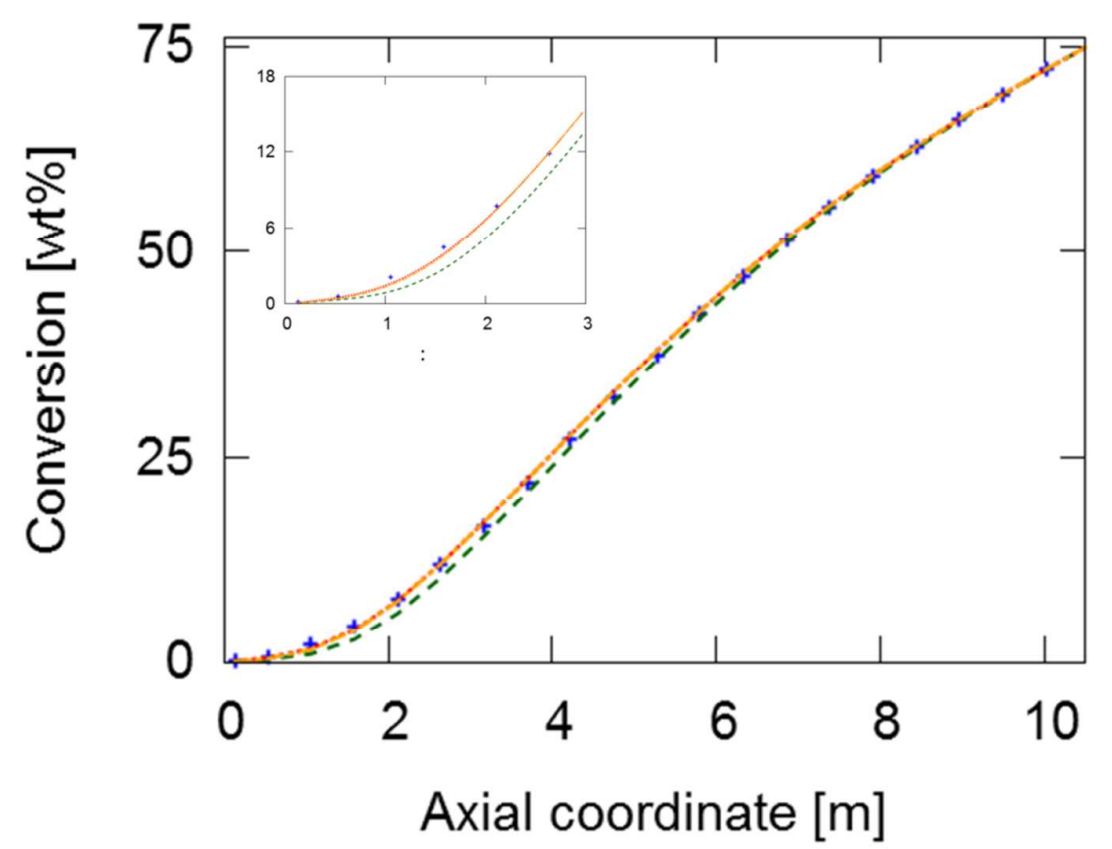

Figure S10: Propane conversion [wt\%] as a function of axial coordinate [m]: + - Bare 3D; - - - - Bare 1D; ....... - Bare 1D T; -- - Bare 1D T, $\beta$.

Besides for ethene and propene, the agreement between the 1D model without accounting for radial profiles and the 3D model for the product yields is satisfactory for the Bare reactor. The propene yield is overpredicted by $0.29 \mathrm{wt} \%$ in the $1 \mathrm{D}$ model and consequently the ethene yield is underpredicted by $0.37 \mathrm{wt} \%$. Accounting for radial profiles of temperature and $\beta$ radicals concentrations slightly improves the agreement but a significant deviation remains. The difference between the 1D model without accounting for radial profiles and the 3D model for the ethene and propene yield is smaller for the SmallFins reactor, i.e. $0.23 \mathrm{wt} \%$ and -0.27 $\mathrm{wt} \%$ respectively. Indeed, the radial temperature and concentration profiles are less pronounced in the SmallFins reactor making the plug flow assumption more accurate for this reactor. When analyzing the effect of the reactor geometry on the product selectivities, it is seen that only for some products the right trends are predicted by the 1D model, even when radial profiles of temperature and $\beta$-radicals are accounted for. The increase in methane and benzene selectivity by application of the SmallFins reactor is well captured. On the contrary, the decrease in ethene selectivity and the increase in propene selectivity are not well captured by the $1 \mathrm{D} \mathrm{T}, \beta$ model. 
Table S5: Comparison simulation results for Bare and SmallFins reactor using a three-dimensional (3D) model and a one-dimensional model without film model (1D), with radial temperature profile (1D T) and with radial temperature and $\beta$-radicals profile (1D T, $\beta$ ).

\begin{tabular}{|c|c|c|c|c|c|c|c|c|}
\hline & Bare 3D & Bare 1D & Bare 1D T & Bare 1D T, $\beta$ & SmallFins 3D & SmallFins 1D & SmallFins 1D T & SmallFins 1D T, $\beta$ \\
\hline Coil-outlet-temperature [K] & 1164 & 1169 & 1168 & 1165 & 1163 & 1168 & 1167 & 1166 \\
\hline Pressure drop [kPa] & 22.9 & 26.7 & 26.6 & 26.6 & 46.8 & 46.7 & 46.6 & 46.7 \\
\hline $\mathrm{P} / \mathrm{E}$ ratio $[\mathrm{wt} \% / \mathrm{wt} \%]$ & 0.73 & 0.75 & 0.75 & 0.75 & 0.74 & 0.75 & 0.75 & 0.75 \\
\hline Propane conversion [-] & 74.2 & 74.2 & 74.2 & 74.2 & 74.2 & 74.2 & 74.2 & 74.2 \\
\hline \multicolumn{9}{|l|}{ Product Yields [wt\%] } \\
\hline C4- & 93.83 & 93.82 & 93.86 & 93.80 & 93.84 & 93.82 & 93.84 & 93.80 \\
\hline [C5+-Benzene] & 2.62 & 2.45 & 2.44 & 2.44 & 2.60 & 2.43 & 2.43 & 2.43 \\
\hline [Benzene-Naphthalene] & 3.56 & 3.74 & 3.70 & 3.76 & 3.56 & 3.74 & 3.73 & 3.76 \\
\hline $\mathrm{H}_{2}$ & 2.03 & 2.02 & 2.01 & 2.02 & 2.01 & 2.01 & 2.01 & 2.01 \\
\hline $\mathrm{CH}_{4}$ & 14.67 & 14.85 & 14.85 & 14.84 & 14.77 & 14.89 & 14.90 & 14.91 \\
\hline $\mathrm{C}_{2} \mathrm{H}_{2}$ & 0.66 & 0.66 & 0.66 & 0.66 & 0.65 & 0.65 & 0.64 & 0.65 \\
\hline $\mathrm{C}_{2} \mathrm{H}_{4}$ & 26.72 & 26.35 & 26.39 & 26.36 & 26.57 & 26.30 & 26.32 & 26.31 \\
\hline $\mathrm{C}_{2} \mathrm{H}_{6}$ & 1.15 & 1.13 & 1.15 & 1.13 & 1.13 & 1.15 & 1.16 & 1.15 \\
\hline $\mathrm{C}_{3} \mathrm{H}_{4}$ & 0.78 & 0.77 & 0.76 & 0.78 & 0.77 & 0.76 & 0.75 & 0.76 \\
\hline $\mathrm{C}_{3} \mathrm{H}_{6}$ & 19.46 & 19.75 & 19.73 & 19.74 & 19.57 & 19.80 & 19.78 & 19.78 \\
\hline $\mathrm{C}_{3} \mathrm{H}_{8}$ & 25.81 & 25.81 & 25.82 & 25.82 & 25.80 & 25.81 & 25.81 & 25.80 \\
\hline $1,3-\mathrm{C}_{4} \mathrm{H}_{6}$ & 1.42 & 1.40 & 1.41 & 1.38 & 1.41 & 1.39 & 1.39 & 1.37 \\
\hline $1-\mathrm{C}_{4} \mathrm{H}_{8}$ & 0.95 & 0.88 & 0.88 & 0.87 & 0.95 & 0.88 & 0.88 & 0.87 \\
\hline $2-\mathrm{C}_{4} \mathrm{H}_{8}$ & 0.13 & 0.13 & 0.13 & 0.13 & 0.13 & 0.13 & 0.13 & 0.13 \\
\hline $\mathrm{i}-\mathrm{C}_{4} \mathrm{H}_{8}$ & 0.06 & 0.06 & 0.06 & 0.06 & 0.06 & 0.06 & 0.06 & 0.06 \\
\hline $\mathrm{n}-\mathrm{C}_{4} \mathrm{H}_{10}$ & 0.01 & 0.01 & 0.01 & 0.01 & 0.01 & 0.01 & 0.01 & 0.01 \\
\hline 1,3-Cyclopentadiene & 0.19 & 1.44 & 1.43 & 1.45 & 0.19 & 1.43 & 1.42 & 1.43 \\
\hline Me-1,3-Cyclopentadiene & 0.36 & 0.32 & 0.31 & 0.32 & 0.36 & 0.32 & 0.32 & 0.32 \\
\hline Benzene & 2.32 & 2.67 & 2.65 & 2.68 & 2.34 & 2.68 & 2.68 & 2.69 \\
\hline Toluene & 0.37 & 0.33 & 0.32 & 0.33 & 0.36 & 0.33 & 0.32 & 0.33 \\
\hline Styrene & 0.09 & 0.08 & 0.08 & 0.08 & 0.09 & 0.08 & 0.08 & 0.08 \\
\hline Naphthalene & 0.78 & 0.67 & 0.66 & 0.67 & 0.77 & 0.66 & 0.66 & 0.67 \\
\hline
\end{tabular}




\begin{tabular}{|c|c|c|c|c|c|c|c|c|}
\hline \multicolumn{9}{|l|}{ Product Selectivity [wt\%] } \\
\hline C4- & 91.68 & 91.66 & 91.73 & 91.64 & 91.70 & 91.67 & 91.69 & 91.65 \\
\hline [C5+-Benzene[ & 3.53 & 3.30 & 3.28 & 3.29 & 3.51 & 3.28 & 3.28 & 3.28 \\
\hline [Benzene-Naphthalene] & 4.79 & 5.04 & 4.99 & 5.06 & 4.79 & 5.05 & 5.03 & 5.07 \\
\hline $\mathrm{H}_{2}$ & 2.73 & 2.72 & 2.71 & 2.73 & 2.72 & 2.71 & 2.70 & 2.71 \\
\hline $\mathrm{CH}_{4}$ & 19.77 & 20.01 & 20.02 & 20.00 & 19.91 & 20.07 & 20.08 & 20.09 \\
\hline $\mathrm{C}_{2} \mathrm{H}_{2}$ & 0.89 & 0.89 & 0.88 & 0.90 & 0.87 & 0.87 & 0.87 & 0.87 \\
\hline $\mathrm{C}_{2} \mathrm{H}_{4}$ & 36.01 & 35.52 & 35.58 & 35.53 & 35.81 & 35.45 & 35.47 & 35.46 \\
\hline $\mathrm{C}_{2} \mathrm{H}_{6}$ & 1.55 & 1.52 & 1.55 & 1.53 & 1.53 & 1.55 & 1.57 & 1.55 \\
\hline $\mathrm{C}_{3} \mathrm{H}_{4}$ & 1.05 & 1.04 & 1.03 & 1.05 & 1.03 & 1.02 & 1.02 & 1.03 \\
\hline $\mathrm{C}_{3} \mathrm{H}_{6}$ & 26.22 & 26.62 & 26.59 & 26.61 & 26.38 & 26.68 & 26.66 & 26.65 \\
\hline $1,3-\mathrm{C}_{4} \mathrm{H}_{6}$ & 1.91 & 1.88 & 1.90 & 1.86 & 1.89 & 1.87 & 1.88 & 1.85 \\
\hline $1-\mathrm{C}_{4} \mathrm{H}_{8}$ & 1.28 & 1.18 & 1.19 & 1.17 & 1.28 & 1.18 & 1.18 & 1.17 \\
\hline $2-\mathrm{C}_{4} \mathrm{H}_{8}$ & 0.17 & 0.17 & 0.17 & 0.17 & 0.17 & 0.17 & 0.17 & 0.17 \\
\hline $\mathrm{i}-\mathrm{C}_{4} \mathrm{H}_{8}$ & 0.09 & 0.08 & 0.08 & 0.08 & 0.09 & 0.08 & 0.08 & 0.08 \\
\hline $\mathrm{n}-\mathrm{C}_{4} \mathrm{H}_{10}$ & 0.01 & 0.01 & 0.01 & 0.01 & 0.01 & 0.01 & 0.01 & 0.01 \\
\hline 1,3-Cyclopentadiene & 0.26 & 1.95 & 1.92 & 1.95 & 0.26 & 1.92 & 1.91 & 1.93 \\
\hline Me-1,3-Cyclopentadiene & 0.48 & 0.43 & 0.42 & 0.42 & 0.48 & 0.42 & 0.42 & 0.42 \\
\hline Benzene & 3.12 & 3.59 & 3.57 & 3.61 & 3.15 & 3.61 & 3.61 & 3.63 \\
\hline Toluene & 0.50 & 0.44 & 0.44 & 0.45 & 0.49 & 0.44 & 0.44 & 0.44 \\
\hline Styrene & 0.12 & 0.11 & 0.10 & 0.11 & 0.12 & 0.10 & 0.10 & 0.10 \\
\hline Naphthalene & 1.05 & 0.90 & 0.88 & 0.90 & 1.04 & 0.89 & 0.89 & 0.90 \\
\hline
\end{tabular}




\section{References}

1. Dijkmans, T.; Pyl, S. P.; Reyniers, M.-F.; Abhari, R.; Van Geem, K. M.; Marin, G. B., Production of bio-ethene and propene: alternatives for bulk chemicals and polymers. Green Chem. 2013, 15, 3064-3076.

2. Schietekat, C. M.; Van Cauwenberge, D. J.; Van Geem, K. M.; Marin, G. B., Computational Fluid Dynamics-Based Design of Finned Steam Cracking Reactors. AlChE J. 2014, 60, 794-808.

3. Fatunla, S. O., Numerical methods for initial value problems in ordinary differential equations. Academic Press Professional, Inc.: 1988; p 295.

4. Felden, A. Review of kinetic reduction techniques available with KINALC; 2013.

5. Turanyi, T.; Tomlin, A. S.; Pilling, M. J., On the error of the quasi-steady-state approximation. J. Phys. Chem. 1993, 97, 163-172.

6. Golub, G. H.; Van Loan, C. F., Matrix-computations. North Oxford Academic: Oxford, 1983; p xvi, 476 p.

7. Noschese, S.; Pasquini, L., Eigenvalue condition numbers: Zero-structured versus traditional. J. Comput. Appl. Math. 2006, 185, 174-189.

8. Wilkinson, J. H., Rigorous Error-Bounds for Computed Eigensystems. Comput J 1961, 4, 230-\&.

9. Wilkinson, J. H., The algebraic eigenvalue problem. Oxford University Press, Inc.: 1988.

10. Demmel, J. W., Applied numerical linear algebra. Society for Industrial and Applied Mathematics: Philadelphia, 1997; p xii, 419 p.

11. Van Geem, K. M.; Reyniers, M. F.; Marin, G. B., Challenges of modeling steam cracking of heavy feedstocks. Oil Gas Sci. Technol. 2008, 63, 79-94.

12. Colebrook, C. F., Turbulent Flow In Pipes, With Particular Reference To The Transition Region Between The Smooth And Rough Pipe Laws. J. Inst. Civ. Eng. 1939, 11, 133 156.

13. Prandtl, L., Mechanics of Viscous fluids. In Aerodynamic Theory, Springer-Verlag: Berlin, 1935.

14. Gnielinski, V., New equations for heat and mass transfer in turbulent pipe and channel flow. Int. Chem. Eng. 1976, 16, 359-368.

15. Van Geem, K. M.; Heynderickx, G. J.; Marin, G. B., Effect of radial temperature profiles on yields in steam cracking. AlChE J. 2004, 50, 173-183.

16. Sundaram, K. M.; Froment, G. F., A comparison of simulation models for empty tubular reactors. Chem. Eng. Sci. 1979, 34, 117-124. 